\title{
An Orexin Hotspot in Ventral Pallidum Amplifies Hedonic 'Liking' for Sweetness
}

\author{
Chao-Yi Ho*,' and Kent C Berridge*, \\ 'Department of Psychology, University of Michigan, Ann Arbor, MI, USA
}

\begin{abstract}
Orexin (hypocretin) is implicated in stimulating appetite as well as arousal, and in both food reward and drug reward. The ventral pallidum (VP) receives orexin projections from lateral hypothalamus neurons (LH), and orexin terminals are especially dense in the posterior half of VP, which is also the location of an opioid hedonic hotspot. The VP hotspot is a roughly cubic-millimeter site where mu opioid stimulation can amplify the hedonic impact of sweetness, expressed as an increase in 'liking' reactions to sucrose taste. The anatomical overlap in posterior VP between opioid hotspot and orexin inputs raises the possibility that the hedonic hotspot might allow orexin to amplify 'liking' too. We examined whether microinjections of orexin-A into the VP hotspot enhance the hedonic impact of sucrose, as assessed via affective taste reactivity measures of 'liking' reactions, and additionally compared effects at nearby sites in adjacent $\mathrm{LH}$ and extended amygdala. Taste reactivity results indicated that orexin stimulation specifically in the VP hotspot nearly doubled the magnitude of positive 'liking' reactions elicited by the taste of sucrose. Mapping results for localization of function, aided by Fos plume measures of the local spread of orexin impact, suggested that hedonic enhancement was generated by essentially the same cubicmillimeter of posterior VP previously identified as the opioid hotspot. By contrast, microinjection sites in the anterior half of VP, or in LH or extended amygdala, generally failed to produce any hedonic enhancement. We conclude that an orexin hedonic hotspot exists in posterior VP, with similar boundaries to the opioid hotspot. An orexin hedonic hotspot may permit regulatory hypothalamic circuitry to make foods more 'liked' during hunger by acting through VP. Dysfunction in a VP orexin hotspot in addiction or mood disorders might also contribute to some types of affective psychopathology.

Neuropsychopharmacology (20 I3) 38, 1655-1664; doi: I0.1038/npp.20 I3.62; published online 3 April 2013
\end{abstract}

Keywords: ventral pallidum; lateral hypothalamus; pleasure; addiction; reward; eating disorders

\section{INTRODUCTION}

Orexin neurons in lateral hypothalamus (LH) are implicated in food reward and hunger, as well as in motivation for nonfood rewards including addictive drugs (Aston-Jones and Harris, 2004; Zheng et al, 2007; Aston-Jones et al, 2010; Choi et al, 2010; Jupp et al, 2011; España and Calipari, 2012). Orexin neurons from $\mathrm{LH}$ project to many forebrain targets, including the ventral pallidum (VP) (Baldo et al, 2003). Within the VP, orexin inputs are especially dense in its posterior half (Baldo et al, 2003). Correspondingly, VP neurons express orexin-A and orexin-B receptors (Marcus et al, 2001), and send reciprocal output projections back to the LH (Groenewegen et al, 1993; Haber et al, 1993).

The posterior VP also contains a specialized anatomical node for enhancing sensory pleasure, in the form of a hedonic hotspot. The hedonic hotspot is a cubic-millimeter subregion of VP where mu opioid microinjection stimulation enhances the positive hedonic impact of sucrose taste

\footnotetext{
*Correspondence: Dr KC Berridge or Dr C-Y Ho, Department of Psychology, University of Michigan, 530 Church Street, Ann Arbor, MI 48 I09-1043, USA. Tel: +7347634365, E-mail: berridge@umich.edu or chaoyiho@gmail.com

Received 26 November 2012; revised 12 January 2013; accepted 15 January 2013; accepted article preview online 5 March 2013
}

(Peciña and Berridge, 2005; Smith and Berridge, 2005; Shimura et al, 2006; Mahler et al, 2007; Smith and Berridge, 2007; Smith et al, 2009; Wassum et al, 2009; Smith et al, 2011). Such 'liking' enhancements can be measured via increases in positive affective orofacial reactions elicited by sweetness, which are homologous in rats, monkeys, apes, and humans (Steiner, 1973; Grill and Norgren, 1978; Berridge, 2000; Steiner et al, 2001). For example, microinjection of the mu opioid agonist (DAMGO) into the posterior VP hotspot can more than double the number of positive 'liking' reactions elicited by sucrose tastes(Peciña and Berridge, 2005; Smith and Berridge, 2005; Mahler et al, 2007; Ho, 2010).

The projection of orexin neurons from LH into the hedonic hotspot of posterior VP suggests the possibility that the VP hotspot might also mediate orexin-induced enhancement of the hedonic impact of foods. Ever since Aristotle it has been known that foods taste better when hungry than when satiated (Aristotle 350BC/2009; Cabanac, 1971);(Cabanac, 1971; Kringelbach, 2005). Increased sensory pleasure of food in hunger compared with satiety has been termed 'alliesthesia' (homeostatic physiological modulation of sensory hedonic impact) (Cabanac, 1971; Kringelbach, 2005). The candidacy of orexin as a mechanism of alliesthesia would become more plausible if orexin in VP were shown to be able to enhance the hedonic 
impact of food reward. Some support for VP participation in hedonic modulation of alliesthesia comes from previous demonstrations that neuronal firing patterns in the VP hotspot encode the increase of hedonic palatability or 'liking' alliesthesia of tastes during physiologically induced natural appetite, and that VP hotspot neurons also encode increases in sucrose palatability induced by pharmacological opioid stimulation of accumbens-pallidum hotspot circuitry (Tindell et al, 2006; Smith et al, 2011; Robinson and Berridge, 2013).

But as yet there is no direct evidence that orexin stimulation of neurons within the VP hotspot can enhance hedonic 'liking' reactions to sucrose taste. We examined whether microinjections of orexin-A into the VP hotspot would magnify sweetness hedonic impact, as assessed by the affective taste reactivity paradigm, and mapped the anatomical boundaries for effective enhancement by also comparing orexin microinjections at sites in other structures, such as LH and extended amygdala. Mapping was aided by estimating the local spread of neurobiological impact of orexin microinjections using a measure of Fos plumes surrounding microinjection sites. Our results support the hypothesis that an orexin hedonic hostpot exists in VP, and indicate the hotspot is localized specifically to the posterior VP without extending to adjacent structures.

\section{MATERIALS AND METHODS}

\section{Surgery}

Under anesthesia $(80 \mathrm{mg} / \mathrm{kg}$ ketamine $\mathrm{HCl}, 10 \mathrm{mg} / \mathrm{kg}$ xylazine, and $0.2 \mathrm{ml}$ atropine sulfate), male SpragueDawley rats $(n=26$ taste reactivity group, $250-400$ g; plus $n=21$ additional rats in Fos plume group) were bilaterally implanted with intracranial microinjection guide cannulae (23 gauge) aimed at $2 \mathrm{~mm}$ above target sites in the VP, or above nearby sites in VP or adjacent structures such as anterior LH or sublenticular extended amygdala (SLEA). Sites were always bilaterally identical within a rat, but individualized coordinates were staggered across rats in both the anterior-posterior plane and medial-lateral plane to cover a range of subregions within VP, and within adjacent LH and SLEA. Coordinates for guide cannula intended to reach VP were: anteroposterior (AP) +0.7 to $-1.2 \mathrm{~mm}$ relative to bregma; mediolateral $(\mathrm{ML})+2.0$ to $-3.2 \mathrm{~mm}$; dorsoventral (DV) -5.8 to $-6.4 \mathrm{~mm}$. Coordinates for LH and SLEA were: AP -1.4 to $-2.5 \mathrm{~mm} ; \mathrm{ML} \pm 1.6 \sim 2.6 \mathrm{~mm} ; \mathrm{DV}-5.7$ to $-6.5 \mathrm{~mm}$. Microinjector tip projected $2.5 \mathrm{~mm}$ beyond the guide cannula. Actual anatomical sites of microinjector tips were assessed histologically after the experiment. Cannulae were anchored to the skull with stainless steel screws and dental cement. Obturators were inserted in guide cannulae to prevent clogging.

In the same surgery, all rats were bilaterally implanted with intraoral cannulae (PE-100 tubing) for delivery of the taste solutions. All rats were given at least 7 days to recover before the first test day. Rats had constant free access to food and water in their home cage, and were never food deprived.

\section{Drugs and Microinjections}

Orexin-A (also known as hypocretin 1) was chosen for microinjection because this ligand binds to both OxR1 orexin receptors (also known as HcrtR1 receptors) and OxR2 receptors (also known as HcrtR2 receptors). Both orexin receptors are found in the VP region of basal forebrain (Marcus et al, 2001), and OxR1 receptors in particular have been implicated in reward seeking behavior (Aston-Jones et al, 2010). Orexin-A (10 nM in $0.2 \mu \mathrm{l} \mathrm{ACSF}$ ) was dissolved in ACSF (dose selected to be within the range able to elicit increases in short-term food intake at subcortical limbic sites and promote seeking of nonfood rewards) (Harris et al, 2005; Thorpe and Kotz, 2005). Within-subject comparisons were used to contrast microinjection effects of orexin $v s$ vehicle (control) in the same rats on reactivity to sucrose (tested twice on separate days: vehicle $v s$ orexin) and quinine (also tested twice, described below). Microinjection order of orexin and vehicle was counterbalanced across rats and all tests were spaced at least $48 \mathrm{~h}$ apart. On a particular test day, each rat received bilateral microinjections of $0.2 \mu \mathrm{l}$ per side of either orexin $10 \mathrm{nM}$ or vehicle. Microinjector tips were held in place for an additional $1 \mathrm{~min}$ after infusion to allow drug to diffuse away from the cannula tip and to avoid backflow. After microinjections, obturators were inserted back to the cranial cannulae, and rats were placed into the transparent taste reactivity chamber with intraoral fluid delivery tubing attached (described in behavioral taste reactivity testing).

\section{Behavioral Taste Reactivity Testing}

The general procedure of behavioral testing is shown in Figure 1. Rats were handled and habituated in the taste reactivity chamber for $30 \mathrm{~min}$ on 4 consecutive days before behavioral testing began, and received a mock microinjection of vehicle on the final habituation day. The other end of the tubing was attached to a syringe pump, which infuses solutions at a stable rate of $1 \mathrm{ml} / \mathrm{min}$. To elicit positive orofacial reactions, all rats received an oral infusion of sucrose solution $(1 \%$ or $0.03 \mathrm{M}, 1 \mathrm{ml}$ volume infusion in $1 \mathrm{~min}$ duration) that began $30 \mathrm{~min}$ after orexin/vehicle microinjection. A separate taste reactivity test of aversive reactions using an oral infusion of quinine sulfate solution as stimulus $\left(3 \times 10^{-4} \mathrm{M}, 1 \mathrm{ml}\right.$ in $\left.1 \mathrm{~min}\right)$ was given $10 \mathrm{~min}$ after the sucrose infusion to 9 rats in order to compare orexin effects on disgust (40 min after microinjection). Quinine was always infused after sucrose, because we were primarily interested in positive-valence hedonic 'liking' responses to sucrose, which may be more vulnerable to contamination from the prior taste than robust aversive disgust responses to quinine, and we wished to avoid any undue suppression of positive hedonic reactions by immediately prior bitterness. Orofacial hedonic and aversive reactions to taste were video recorded for subsequent analysis.

\section{Taste Reactivity Video Scoring}

Hedonic, aversive, and neutral patterns of taste reactivity were scored offline using frame-by-frame slow-motion videotape analysis (Berridge, 2000). Positive hedonic 


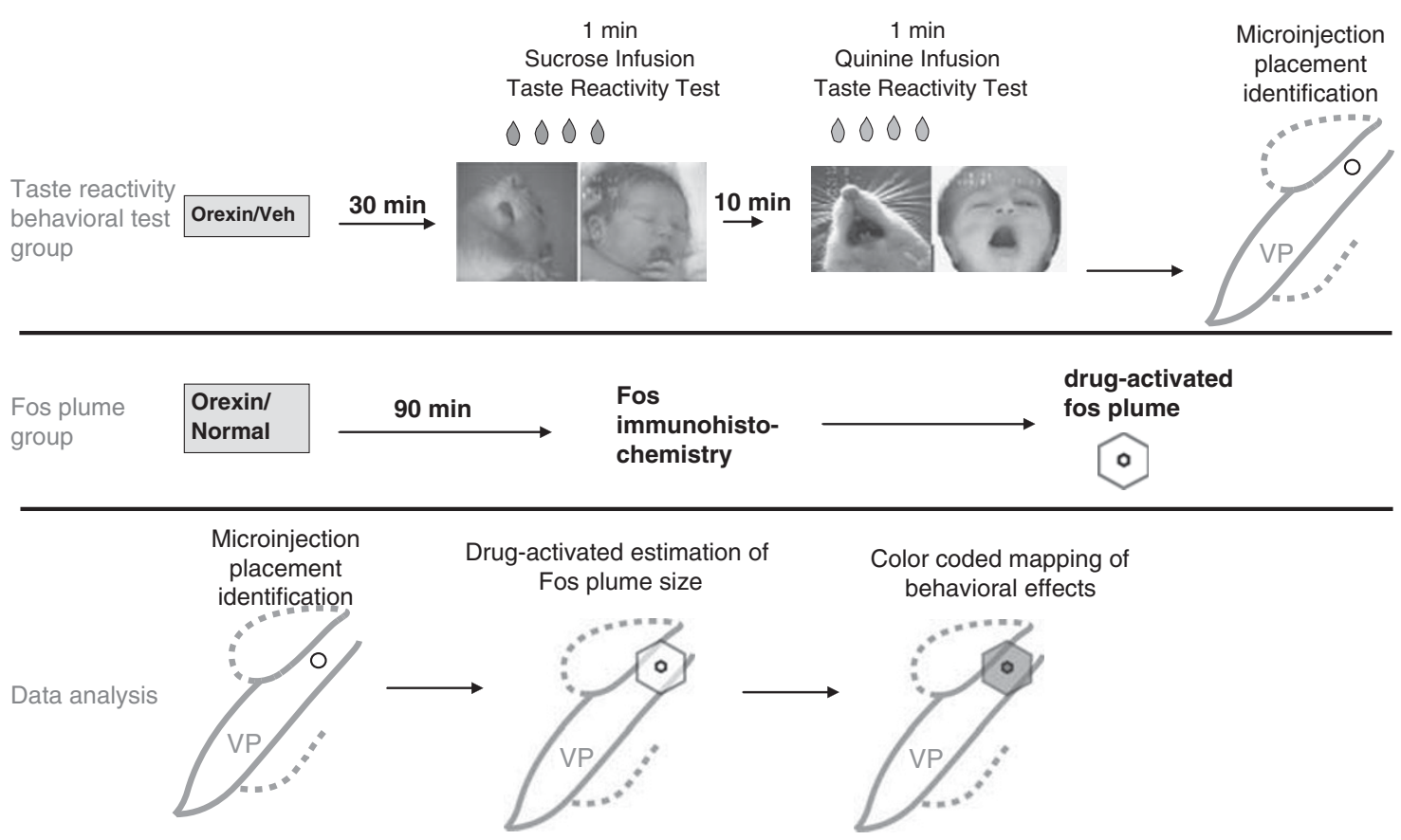

Figure I Procedure for mapping localization of function. Logic of the mapping procedure used to identify the functional hotspot is shown. The top two levels depict the two groups constituting the 'split and recombine' steps needed to identify the neural substrate responsible for hedonic enhancement. The top row shows procedures for the behaviorally tested group that produced taste reactivity data. The second row shows procedures for the Fos plume group that produced neural spread of impact data. The third row shows how data sets from both groups were recombined to create function maps: the size of sympols was determined by Fos plume data, whereas the placement and color of symbols were determined by plotting taste reactivity and site data from the behavioral group.

responses included rhythmic tongue protrusions, lateral tongue protrusions, and paw licks. Aversive responses included gapes, headshakes, face washes, forelimb flails, and chin rubs. Neutral responses, which are less consistently linked to hedonic/aversive taste valuation, included passive dripping of solution out of the mouth and rhythmic mouth movements evoked by oral infusions (grooming and doing nothing were also considered neutral reactions). All video analyses were conducted blind to the microinjection condition and cannula placements using Observer software (Noldus, Netherlands).

A time-bin scoring procedure was used to quantify hedonic and aversive orofacial components. All video analyses were conducted using Observer software by an observer blind to drug/vehicle condition and to cannulae placement. A time-bin scoring procedure was used to ensure that taste reactivity components of different relative frequency were balanced in their contributions to the final affective hedonic/aversive totals (Berridge, 2000). For example, rhythmic mouth movements, passive dripping of sucrose or quinine solution, paw licking, and grooming behaviors typically occur in long bouts, and were thus scored in $5 \mathrm{~s}$ time bins (up to $5 \mathrm{~s}$ continuous bout duration equaled one occurrence). Rhythmic tongue protrusions along the midline, which occur in shorter bouts, were scored in $2 \mathrm{~s}$ time bins. The other behavioral components (lateral tongue protrusions, gapes, forelimb flails, headshakes, chin rubs) typically occur as discrete events and were therefore scored as single occurrences each time they appeared (eg, one gape scored as one occurrence).

Individual totals were calculated for hedonic reactions for each rat by adding all positive response scores for that rat. A separate total for aversive reaction scores was calculated similarly for each rat. Changes in taste reactivity induced by orexin were calculated as a percent change in positive hedonic total score for each rat by comparison to the same rat's vehicle control score for statistical comparisons and function map of Figure 3, or by substraction of vehicle score from orexin score for statistical comparisons and the bar graph of Figure 4.

\section{Histology and Construction of Fos Plume Map for Localization of Function}

In order to map how far orexin microinjections spread to impact neurons in local tissue, and help assign anatomical responsibility for orexin-induced hedonic enhancements to responsible neural substrates, we used a Fos plume tool that measures local plume-shaped regions of neuronal Fos modulation caused by microinjections. Local plumes of Fos expression provide a relatively direct measure of the spread of drug impact on local brain tissue, in the form of immediate early gene transcription and translation within nearby neurons. Fos plumes are localized regions where Fos protein expression is modulated immediately surrounding a drug microinjection (compared with a normal brain or to vehicle microinjections). The radius of a Fos plume indicates the extent of spread of impact on local neurons of drug contained in a microinjection, at least in terms of altering immediate early gene $c$-fos transcription and translation into protein.

However, the radius of a Fos plume can shrink after a series of microinjections in the same location, just as behavioral effects of drug microinjections can decline after 

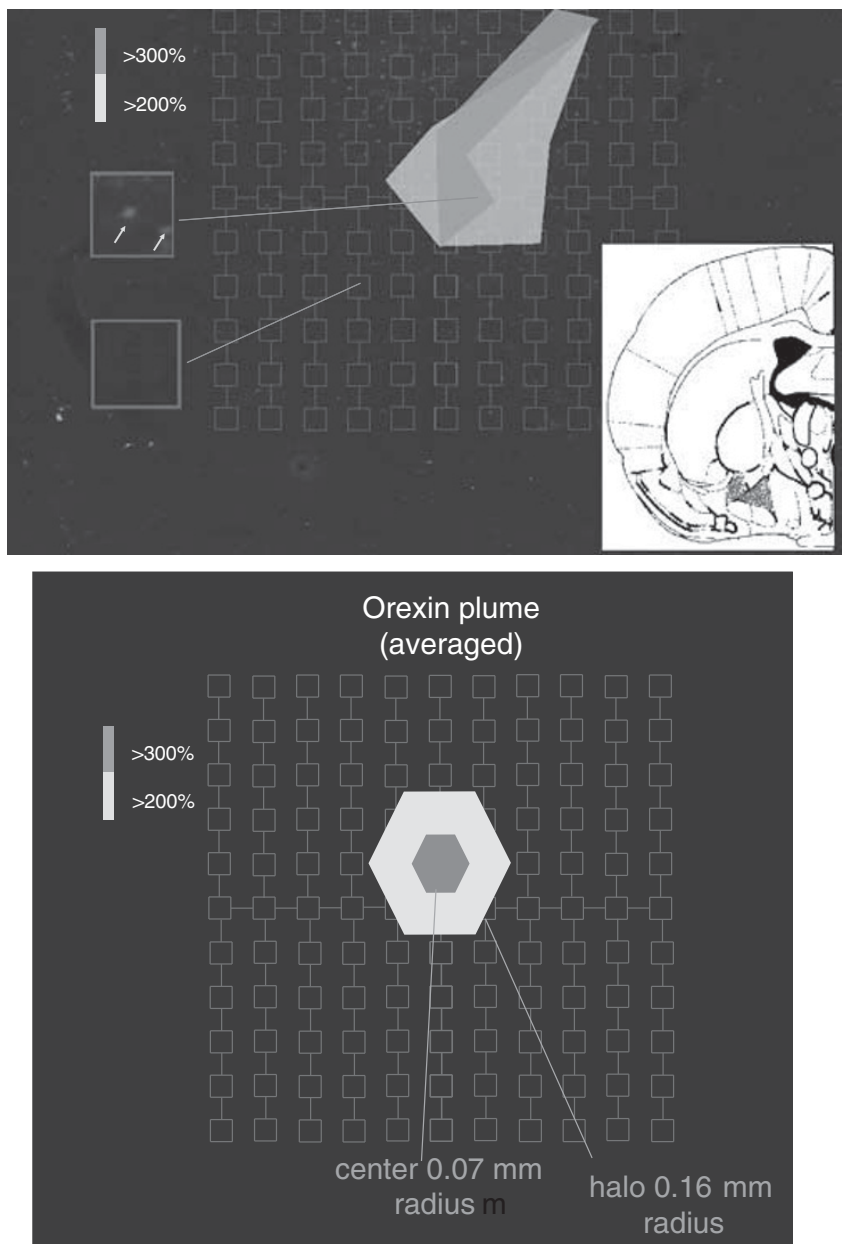

Figure 2 Orexin Fos plume examples. A sample Fos plume produced by orexin microinjection is shown at top. Fos-expressing neurons were counted in $50 \times 50 \mu \mathrm{m}^{2}$ blocks (shown as squares) on eight sampling arms (extending from the center of microinjection center at $50 \mu \mathrm{m}$ intervals. Normal Fos expression was I-2 neurons per block, but orexin microinjection elevated Fos expression up to 3-6 neurons per block). The color scale shows the intensity of Fos elevation (over control levels; $200 \%=$ yellow and $300 \%=$ orange). The averaged plume diameter from all orexin microinjections, which was used to determine the size of mapping symbols in Figures 3 and 4, is shown at bottom.

a series of several, presumably due to gradual accrual of gliosis and necrosis that limits subsequent drug impact (Richard and Berridge, 2011). Shrinkage of a final microinjection plume could lead to false inference that the diameter of impact was smaller than it actually had been on an earlier microinjection. To avoid plume shrinkage that would result in underestimation of the spread of drug impact and distort inferences about localization of function, we used a split-and-recombine procedure that has been used successfully in previous studies to integrate maximum Fos plume diameter and microinjection effects on behavior into a single map for localization of function (Peciña and Berridge, 2005; Smith and Berridge, 2005; Richard and Berridge, 2011) (Figure 2). This procedure involves measuring behavioral effects of microinjections in one group and measuring anatomical Fos plume radius in a separate group of rats after their first orexin microinjection administered under conditions similar to the behavioral
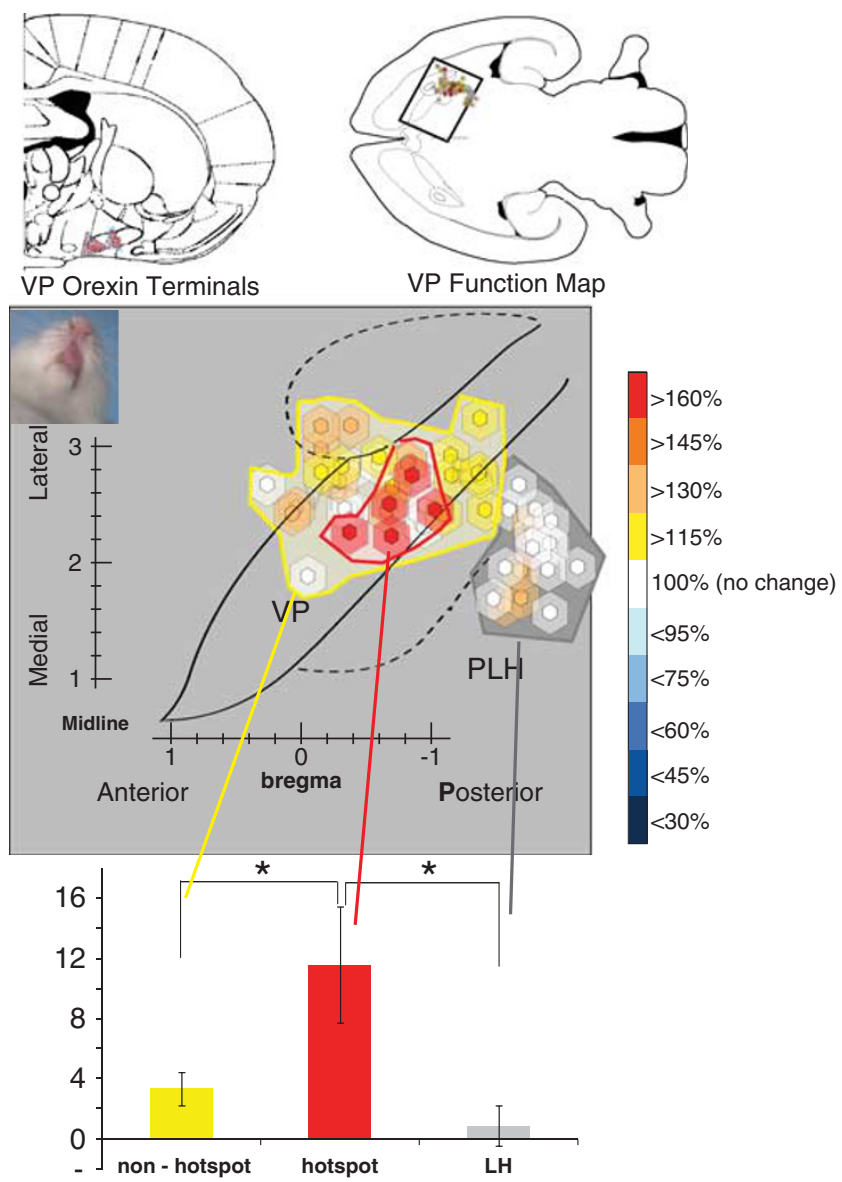

Figure 3 Orexin hotspot in ventral pallidum (VP): function map for hedonic enhancement. Map plots the causation effects for enhancement of sucrose 'liking' reactions in a horizontal map of VP and nearby structures (above). The horizontal plane best shows the relative effectiveness of all sites within the VP (map is blownup from horizontal brain section shown in top right inset). A hedonic hotspot is revealed as by the localized cluster of red symbols in posterior VP, where orexin caused intense hedonic enhancements above 160\% (100\%=vehicle control levels in the same rats). By comparison, non-hotspot sites in an adjacent surrounding band produced much lower enhancements of about I I $5 \%$ above vehicle. Finally, no hedonic enhancement at all was produced by sites further away from the hotspot in VP, or by all sites in the preoptic lateral hypothalamus (white symbols). Bar graphs (bottom) show the average change in score for positive hedonic 'liking' reactions (orexin minus vehicle control scores for each rat) elicited by sucrose after orexin microinjecitions in VP hotspot (red center bar), non-hotspot surrounding band of VP (yellow left bar), or lateral hypothalamus (gray right bar). Error bars represent SEM. *Indicates significant increases in hedonic reactions $(P<0.05)$ of orexin compared with vehicle, within-subject comparison. Top left inset depicts the density of orexin/hypocretin (HCRT)- immunopositive fibers (red dots on gray) in a coronal section of rat VP at a level through the posterior hotspot, redrawn with permission from Baldo et al (2003).

group's testing conditions to ascertain maximal diameters for spread of impact on tissue surrounding a microinjection. Spread is likely to be maximal after the first microinjection received by a rat.

Brain slices from rats used for behavioral testing were processed for cresyl violet to identify the center of the microinjection site and plotted onto a brain atlas (Figure 1). Brain slices from Fos plume rats $(n=7)$ were processed for Fos expression and plume evaluation as previously described (Richard and Berridge, 2011). Only radius-of-spread 

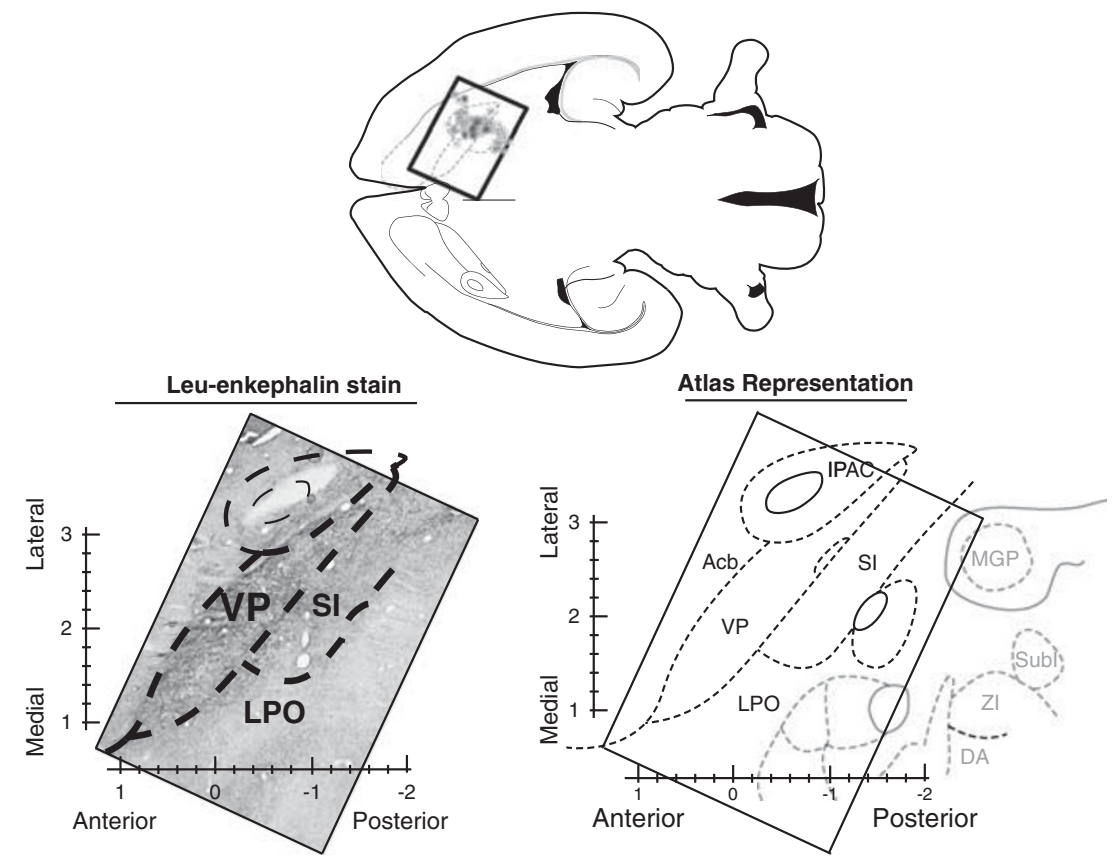

VP Hotspot Comparison Map

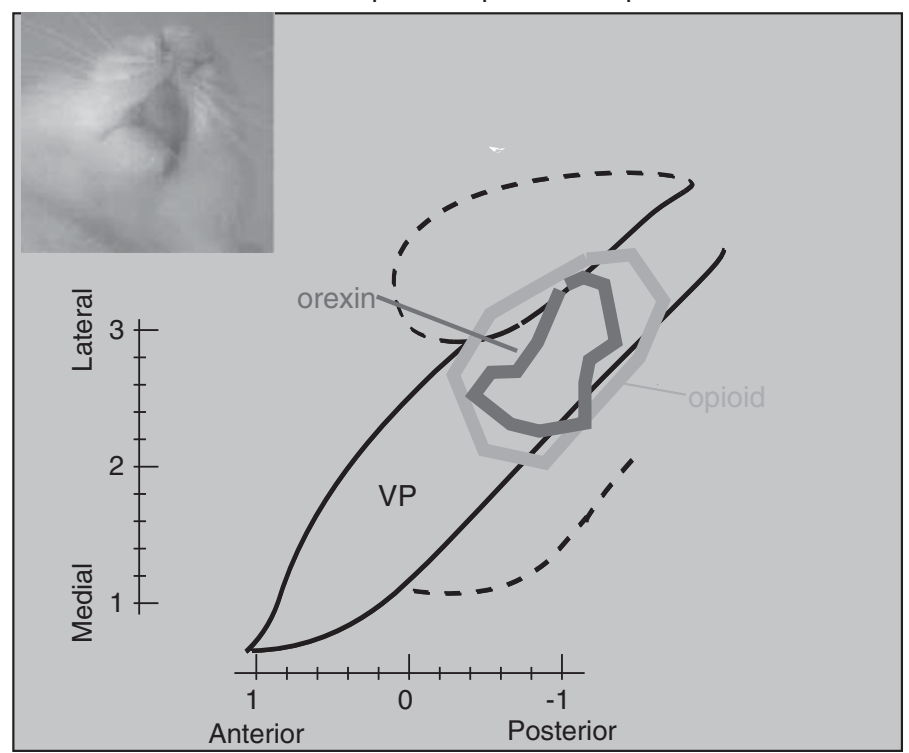

Figure 4 Comparison of orexin hotspot to opioid hedonic hotspot in ventral pallidum (VP). Horizontal brain shown at top. Second row shows blowup of VP visible in horizontal section stained by leu-enkephalin (modified from Smith and Berridge (2005)). Corresponding atlas representation from Paxinos and Watson (1998) at right. Bottom shows a comparison of orexin hotspot identified here in caudal VP (red line) relative to the mu opioid hedonic hotspot (orange line) mapped previously from Smith and Berridge (2005). The color reproduction of this figure is available on the Neuropsychopharmacology online.

data were obtained from Fos plume measurements, and the measured radii of Fos plumes were used solely to assign the size of symbols in maps (Figure 1). All other data in maps were obtained from behaviorally tested rats: each individual's data were used to assign the hedonic consequence (symbol color) and microinjection site (symbol location) of its orexin microinjection (orexin microinjection hedonic score was compared with vehicle microinjection score in the same behaviorally tested rat; bilateral cannulae for each rat were plotted separately). Behavioral changes caused by orexin microinjections were mapped as color plumes onto the identified sites to create hedonic causation maps. The color-coded symbols in the map display the functional causation of taste reactivity changes induced by orexin microinjections, in terms of the consequences on enhancement of positive hedonic reactions to sucrose.

\section{Fos Plume Histology}

Brains were sliced at $40 \mu \mathrm{m}$ on a freezing microtome, and processed for Fos-like immunoreactivity using NDS, goat anti-c-fos (Santa Cruz Biotechnology, Santa Cruz, CA) and donkey anti-goat Alexa Fluor 488 (Invitrogen, Carlsbad, CA). 
Sections were mounted, air-dried, and coverslipped with ProLong Gold antifade reagent (Invitrogen).

Immunoreactivity for Fos-like proteins was visualized using a Leica microscope equipped for fluorescent microscopy, using a filter with an excitation band at $480-505 \mathrm{~nm}$ for Fos-positive cells and images were taken using MCID Core software. For analysis of drug spread, Fos plumes images were taken in the areas surrounding the microinjection with the most intense areas of Fos expression, just medial to the end of the injector tip, surrounding a small focal point of necrosis. Fos labeled cells were individually counted within successive blocks $\left(50 \times 50 \mu \mathrm{m}^{2}\right)$, along eight radial arms emanating from the center of the necrosis, with $\times 10$ magnification.

\section{Statistical Analyses}

Behavioral statistical analyses were two-tailed and $\alpha$ was always set at $P<0.05$. Between-subjects ANOVAs were used to compare anatomical location effects of microinjection sites (eg, VP vs LH, hotspot $v s$ non-hotspot), followed by Bonferroni's post-hoc comparisons. Within- subject paired $t$-tests were used to test orexin $v s$ vehicle effects on behaviors.

\section{RESULTS}

\section{Fos Plume Mapping: Identifying Zones of Local Neuronal Activation}

In terms of anatomical spread of drug impact, Orexin-A microinjections $(10 \mathrm{nM} 0.2 \mu \mathrm{l})$ produced elevations in Fos immediately surrounding the microinjection cannula tip of $\sim 0.3-0.4 \mathrm{~mm}$ diameter (compared with control brain levels of expression). The maximum spherical plume of orexininduced Fos elevation was roughly $0.2 \mathrm{~mm}^{3}$ volume, containing two concentric inner intense and outer moderate zones of Fos enhancement (Figure 2). An inner center radius of $0.065 \pm 0.006 \mathrm{~mm}$ had intense tripled elevation of Fos protein expression surrounding the microinjection site after orexin microinjection, $>300 \%$ above control 'normal' levels that occurred spontaneously in VP. An outer moderate zone of doubled elevation $(>200 \%$ but $<300 \%$ ) of Fos above control levels had a larger $0.16 \pm 0.01 \mathrm{~mm}$ radius. The overall $0.16 \mathrm{~mm}$ radius of the outer Fos plume indicates the approximate size of a maximal sphere of local neuronal impact induced by orexin microinjections. Although it is unknown whether Fos-expressing neurons had orexin receptors themselves or instead were indirectly modulated by receptor-bearing neighbors, the roughly $0.2 \mathrm{~mm}$ volume gives at least some objective information on orexin's spread of local impact. Here, the $0.2 \mathrm{~mm}$ volume (0.016 radius) of maximum impact spread was selected to set the size of mapping symbols in hedonic causation maps to identify hotspots. All other data depicted in maps (colorcoded behavioral intensities, bars, anatomical locations of effective sites) depict functional consequences or orexin microinjection effects on hedonic reactions observed in behaviorally tested rats.

\section{Orexin Hedonic Hotspot in Posterior VP}

Infusions of sucrose solutions into the mouth always elicited positive hedonic ('liking') reactions (eg, rhythmic licking via midline tongue protrusions, paw licks), with nearly zero levels of negative disgust or 'disliking' reactions (eg, gapes, headshakes). However, the magnitude or number of positive hedonic reactions elicited by sucrose varied depending on whether a rat had an orexin microinjection or a vehicle microinjection just before the taste reactivity test, as well as upon the precise anatomical site of orexin microinjection.

Our main finding was that orexin-A microinjections in a limited subregion or hotspot of posterior VP strongly enhanced hedonic impact. Orexin microinjections in this posterior subregion of VP of nearly doubled the number of positive hedonic reactions elicited by sucrose taste (vehicle level $\left.=100 \% ; t_{6}=-2.99, P=0.02 ; n=7\right)$. Enhancement of positive reactions was observed only when sucrose was infused in the mouth, and not before in the absence of taste stimulus. The stimulus dependency suggests that orexin microinjections in posterior VP magnified the hedonic impact of sucrose taste, rather than simply promoting positive orofacial reactions as simple motor responses or inducing a global sustained state of hedonic reactivity independent of stimulus.

Mapping results indicated that an anatomical cluster of sites in posterior VP appeared responsible for essentially all cases of enhanced positive hedonic 'liking' reactions (Figure 3). The anatomical clustering of hedonic enhancement sites was located in a volume of about $1 \mathrm{~mm}^{3}$ in posterior VP, depicted by the group of red plumes in Figure 3 (denoting sites which produced $>150 \%$ enhancement in the number of positive hedonic reactions elicited by the sucrose taste). This contiguous group of 'most effective' sites will be referred to as the VP orexin hotspot in descriptions below. The magnitude of orexin-induced increase averaged $>175 \%$ for this hostpot cluster, compared with vehicle control levels measured in the same rats. By contrast to positive hedonic enhancement of 'liking' reactions, orexin microinjections never altered the very low number of aversive 'disgust' reactions for sucrose, which remained near zero, even in the VP hotspot. Similarly, the higher levels of negative disgust reactions elicited by bitter quinine infusions also remained unchanged by orexin microinjection, as described below, indicating that orexin dselectively enhance the positive hedonic impact of a palatable taste.

Anatomical details of orexin hotspot. The center of anatomical hotspot in VP for hedonic enhancement was at $\sim 0.7 \mathrm{~mm}$ posterior to bregma in the anterior-posterior dimension (extending from -0.2 to -0.9 ), $2.4 \mathrm{~mm}$ lateral to bregma in the ML dimension (extending from 2.2-3.0), and $-7.7 \mathrm{~mm}$ ventral to the skull in the dorsal-ventral dimension (ranging from -7.3 to -8.0 ; mapped in horizontal stereotaxic atlas plane to allow view of nearly entire VP; Figures 3 and 4). At its center, the VP hotspot was positioned ventral to anterior commissure, lateral to substantia innominata and lateral preoptic area, and medial to the interstitial nucleus of the posterior limb of the anterior commissure (a component of extended amygdala). 
The rostral boundary of the hedonic hotspot extended forward to nearly the A-P center of VP (AP $-0.2 \mathrm{~mm}$ to bregma). Caudally, the hotspot extended to the caudal edge of VP (AP $-1.1 \mathrm{~mm}$ ), where VP is lateral to the anterior tip of the $\mathrm{LH}$, and adjacent to the most anterior part of the SLEA.

Sites outside the VP hotspot. Much milder hedonic enhancements of approximately only $15 \%$ over vehicle levels were produced just outside the VP hotspot by orexin microinjections at sites in a surrounding band about $1 \mathrm{~mm}$ wide (Figure 3). Some portion of particular orexin microinjections within the surrounding band might well have penetrated into VP hotspot itself (based on Fos plume diameters above). Orexin microinjections had no detectable effect at all on hedonic sucrose reactions at sites outside the 1-mm band. Ineffective sites included placements rostral to the hotspot in anterior VP. Ineffective sites also included virtually external structures outside VP, such as sites in anterior LH and the lateral preoptic region (caudal-medial to the VP hotspot). Also ineffective were sites in SLEA or substantia innominata (caudal to the hotspot). Explicit comparison of the VP hotspot to those other sites indicated that the enhancement of hedonic reactions was reliably statistically higher inside the VP hotspot (SLEA $P=0.048$; LH $P=0.015$; Figure 3 ).

\section{Orexin Fails to Modulate Aversive 'Disliking' Reactions to Quinine}

Oral infusions of bitter quinine sulfate solution always elicited a disgust pattern of many aversive reactions (eg, gapes) and hardly any positive hedonic reactions. The magnitude of aversive reaction scores for quinine were not altered by orexin microinjections (compared with control scores for vehicle microinjections in the same rats). Even inside the posterior VP hotspot, microinjections of orexin failed to alter aversive reactions (VP hotspot vehicle: $51.8 \pm 11.8$ aversive reactions; orexin: $44.14 \pm 8.4$ aversive reactions, $P=0.61$, NS). Similarly, all outside sites in LH, globus pallidus or extended amygdala failed to alter the number of aversive 'disliking' reactions elicited by quinine (NS). Orexin similarly failed to change the very low level of positive reactions elicited by bitter quinine, whether inside or outside the VP hotspot. Positive reactions to quinine always stayed near zero, suggesting again that orexin hedonic enhancement effects were restricted to amplifying positive impact of an already-pleasant gustatory sensation, such as sucrose, and did not extend to modulating the extreme unpleasantness of bitterness.

\section{DISCUSSION}

\section{Orexin Enhanced 'Liking' Reactions to Sucrose in the Posterior VP}

Overall, our results indicated that a localized anatomical hotspot for orexin-induced hedonic enhancement of sweetness exists in in posterior VP. Within the VP hotspot, orexin microinjections enhanced positive hedonic or 'liking' reactions elicited by the sweet taste of sucrose by over $175 \%$ (eg, lip and paw licking; compared with vehicle control levels), without altering negative 'disliking' reactions elicited by bitter quinine (eg, gapes). The volume of the orexin hedonic hotspot in VP appeared to be $\sim 1 \mathrm{~mm}^{3}$ volume, with a size and boundary similar to those previously defined for the VP opioid hotspot (Figure 4) (Smith and Berridge, 2005; Kupchik and Kalivas, 2012). Strikingly, the orexin hotspot did not seem to extend outside VP to LH, preoptic area, or extended amygdala, as discussed below.

\section{Orexin in LH or SLEA does not Amplify Hedonic 'Liking'}

It is of special interest that the orexin hedonic hotspot did not penetrate the contiguous LH (immediately caudal and medial to VP) despite the facts that orexin neurons originate in the LH (Harris et al, 2005; Aston-Jones et al, 2010), and that LH has well-known roles in promoting food intake (Valenstein et al, 1970; Gao and Horvath, 2007; Berthoud and Morrison, 2008). Nor did the orexin hotspot extend into the contiguous SLEA. Microinjection sites in both LH and SLEA essentially failed to produce detectable hedonic enhancement (Figure 3). Failure to enhance hedonic 'liking' does not exclude orexin or other signals in LH or SLEA from playing direct roles in other aspects of hunger, including enhancing incentive properties of food motivation ('wanting'). Still, our results suggest that in order to magnify hedonic 'liking', orexin signals may need to be conveyed outside LH origin to specialized hedonicgenerating sites, such as the posterior VP hotspot identified here.

\section{Neurobiological Bases of VP Hedonic Hotspot?}

What is neurobiologically unique about the posterior VP hotspot that could explain its uniqueness in generating hedonic enhancements? Although difficult to answer conclusively yet, at least some unique neurobiological features of the posterior VP have begun to be identified that could be relevant to this question (Thompson and Swanson, 2010; Kupchik and Kalivas, 2012). For example, Kupchik and Kalivas (2012) reported results of a recent anatomical and electrophysiological study of anterior and posterior VP subregions, suggesting that the posterior region containing the hotspot has neurons that are relatively excitable and possess long aspiny dendrites (similar to classic globus pallidus). By comparison, the anterior VP (especially rostromedial quadrant), which does not contain the hotspot, has less-excitable neurons with shorter spiny dendrites (more similar to nucleus accumbens (NAc) neurons). Caudal VP may also receive different afferent projections from rostral VP, and send different efferent projections to distinct anatomical targets (Thompson and Swanson, 2010). Although the functional implications of such neurobiological differences are not yet clear, at least it seems plausible that neurobiological differences do exist between caudal and rostral subregions of VP. Those differences could eventually prove relevant to explaining why the posterior VP subregion possesses the specialized hedonic hotspot (Kupchik and Kalivas, 2012). 


\section{Potential Relations to Orexin Function in Reward and Alliesthesia}

Orexin has been implicated in mediating hunger (Harris et al, 2005; Zheng et al, 2007; Berthoud and Morrison, 2008; Gao and Horvath, 2008; Borgland et al, 2009; Aston-Jones et al, 2010; Choi et al, 2010; Berthoud et al, 2011; Thompson and Borgland, 2011; Mahler et al, 2012), and it seems relevant to speculate that an orexin hedonic hotspot in posterior VP, demonstrated by exogenous stimulation here, might also permit any orexin elevations in VP hotspot that occur endogenously during hunger make foods taste better (alliesthesia). Elevation in endogenous orexin release, triggered by NPY released on LH neurons during hunger, could amplify food hedonic impact just as exogenous microinjections of orexin in the VP hotspot did here.

Anatomically, VP is well suited to interface between hypothalamic hunger signals and accumbens-pallidal mechanisms known to mediate food reward, as VP has extensive reciprocal connections with both LH and NAc (Zahm et al, 1996; Groenewegen et al, 1999; Kalivas and Nakamura, 1999; Heimer et al, 2008; Smith et al, 2009; Haber and Knutson, 2010; Napier and Mickiewicz, 2010; Thompson and Swanson, 2010; Stratford and Wirtshafter, 2012). Those neurobiological features might perhaps also contribute to functional uniqueness for hedonic amplification by the posterior VP hotspot.

We note that the hedonic LH-to-VP alliesthesia mechanism sketched here could exist in parallel with a less direct anatomical route for orexin-induced alliesthesia previously suggested by Kelley et al (2005): namely, a multiple-synapse circuit from LH orexin neurons via thalamus to NAc neurons. Kelley et al (2005) suggested that orexin neurons in LH might project first to activate glutamate neurons in the thalamic paraventricular nucleus, which in turn project to acetylcholine-containing interneurons in the medial shell of NAc. Kelley et al (2005) suggested that acetylcholine neurons in medial shell finally activated adjacent enkephalin-containing medium spiny neurons in nucleus accumbens, thus stimulating enkephalin release to engage $\mu$-opioid receptors. Stimulation of NAc opioid receptors would include neurons within the NAc hotspot in rostrodorsal medial shell. The NAc hotspot similarly enhances sucrose 'liking' reactions in response to $\mu$-opioid stimulation (Peciña and Berridge, 2005) and has its own distinct anatomical connectivity features that may be relevant to its hedonic generation function (Thompson and Swanson, 2010; Zahm et al, 2013). Thus, indirect orexin activation of that LH-NAc circuit could enhance hedonic impact of food, as suggested by Kelley et al (2005), in parallel to the more direct LH-VP alliesthesia mechanism implicated by our results.

\section{Circuit and Clinical Implications}

Orexin has also been implicated in nonfood rewards such as addictive drugs (Harris et al, 2005; Harris and Aston-Jones, 2006; Aston-Jones et al, 2010; Smith et al, 2010b; Kim et al, 2012; Mahler et al, 2012). Involvement in drug rewards raises the possibility that the VP orexin hotspot identified here might also have a role in generating intense drug pleasures, and possibly of other non-ingestive sensory rewards.
Of course, whether for food or other rewards, the VP hotspot cannot act by itself to enhance hedonic impact. In order to enhance 'liking' reactions, stimulation of the VP hotspot must recruit larger mesocorticolimbic circuits that extend throughout much of the brain. This includes reciprocal functional interactions with the other hotspot in NAc (Peciña and Berridge, 2005; Mahler et al, 2007; Smith et al, 2011), in ways that form integrated hedonic NAc-VP circuit, which operates as a single entity (Smith and Berridge, 2007; Smith et al, 2010a; Smith et al, 2011). Our results indicate that the VP reward circuit also is influenced by LH regulatory circuits. That is consistent with previous findings mentioned above, and with VP modulation of neuronal firing to tastes and their associated cues related to a type of alliesthesia (sodium appetite) (Tindell et al, 2006; Tindell et al, 2009). Conceivably, such modulation may occur also for caloric foods that activate the VP (Beaver et al, 2006; Stoeckel et al, 2008).

Clinically, regulatory LH and orexin interactions with VP and related hedonic food reward circuits highlighted here may therefore be relevant to understanding some forms of obesity and binge-eating disorders (Aston-Jones et al, 2010; Choi et al, 2010; Mahler et al, 2012). Such roles for VP in hedonic dysfunction pathological over-consumption might also possibly extend to compulsive pursuits of other rewards, including drug addiction (Mahler et al, 2012).

Although clinical implications remain to be explored, these results are the first specific demonstration to our knowledge that orexin has hedonic generation functions able to amplify 'liking' reactions to a sensory pleasure, or that those hedonic functions may be localized in an anatomical hotspot. This VP hedonic mechanism provides a potential way for regulatory hypothalamic neurochemical signal to act on classical reward circuitry and magnify sweetness hedonic impact. By enhancing 'liking' reactions to a sensory pleasure, this orexin hotspot mechanism in VP is well positioned to mediate natural modulations of positive affect that occur in natural appetites, and potentially to also contribute to psychopathological conditions that may distort positive affect, such as binge-eating, drug addiction, or mood disorders.

\section{ACKNOWLEDGEMENTS}

This research was supported by MH63649 and DA015188 grants from the NIH. We thank Brian Baldo and colleagues for allowing us to redraw parts of Figure 3 inset derived from Baldo et al (2003) Figure 1 panels $g$ and h (orexin terminal distribution in VP), and thank Aaron Garcia for redrawing the inset.

\section{DISCLOSURE}

The authors declare no conflict of interest.

\section{REFERENCES}

Aristotle (350BC) (2009). The Nicomachean ethics. Oxford University Press: Oxford, UK.

Aston-Jones G, Harris GC (2004). Brain substrates for increased drug seeking during protracted withdrawal. Neuropharmacology 47: 167-179. 
Aston-Jones G, Smith RJ, Sartor GC, Moorman DE, Massi L, Tahsili-Fahadan P et al (2010). Lateral hypothalamic orexin/ hypocretin neurons: a role in reward-seeking and addiction. Brain Res 1314: 74-90.

Baldo BA, Daniel RA, Berridge CW, Kelley AE (2003). Overlapping distributions of orexin/hypocretin- and dopamine-betahydroxylase immunoreactive fibers in rat brain regions mediating arousal, motivation, and stress. J Comp Neurol 464: 220-237.

Beaver J, Lawrence A, van Ditzhuijzen J, Davis M, Woods A, Calder A (2006). Individual differences in reward drive predict neural responses to images of food. J Neurosci 26: 5160-5166.

Berridge KC (2000). Measuring hedonic impact in animals and infants: microstructure of affective taste reactivity patterns. Neurosci Biobehav Rev 24: 173-198.

Berthoud H-R, Lenard NR, Shin AC (2011). Food reward, hyperphagia, and obesity. Am J Physiol 300: R1266-R1277.

Berthoud HR, Morrison C (2008). The brain, appetite, and obesity. Annu Rev Psychol 59: 55-92.

Borgland SL, Chang S-J, Bowers MS, Thompson JL, Vittoz N, Floresco SB et al (2009). Orexin A/hypocretin-1 selectively promotes motivation for positive reinforcers. J Neurosci 29: 11215-11225.

Cabanac M (1971). Physiological role of pleasure. Science 173: 1103-1107.

Choi DL, Davis JF, Fitzgerald ME, Benoit SC (2010). The role of orexin-A in food motivation, reward-based feeding behavior and food-induced neuronal activation in rats. Neuroscience 167: 11-20.

España R, Calipari ES (2012). Hypocretin/orexin regulation of dopamine signaling: implications for reward and reinforcement mechanisms. Front Behav Neurosci 6: 54.

Gao Q, Horvath TL (2007). Neurobiology of feeding and energy expenditure. Annu Rev Neurosci 30: 367-398.

Gao Q, Horvath TL (2008). Neuronal control of energy homeostasis. FEBS Lett 582: 132-141.

Grill HJ, Norgren R (1978). The taste reactivity test. I. Mimetic responses to gustatory stimuli in neurologically normal rats. Brain Res 143: 263-279.

Groenewegen HJ, Berendse HW, Haber SN (1993). Organization of the output of the ventral striatopallidal system in the rat: ventral pallidal efferents. Neuroscience 57: 113-142.

Groenewegen HJ, Wright CI, Beijer AV, Voorn P (1999). Convergence and segregation of ventral striatal inputs and outputs. Ann N Y Acad Sci 877: 49-63.

Haber SN, Knutson B (2010). The reward circuit: linking primate anatomy and human imaging. Neuropsychopharmacology 35: 4-26.

Haber SN, Lynd-Balta E, Mitchell SJ (1993). The organization of the descending ventral pallidal projections in the monkey. J Comparative Neurol 329: 111-128.

Harris GC, Aston-Jones G (2006). Arousal and reward: a dichotomy in orexin function. Trends Neurosci 29: 571-577.

Harris GC, Wimmer M, Aston-Jones G (2005). A role for lateral hypothalamic orexin neurons in reward seeking. Nature 437: 556-559.

Heimer L, Van Hoesen GW, Trimble M, Zahm DS (2008). Anatomy of Neuropsychiatry: The New Anatomy of the Basal Forebrain and its Implications for Neuropsychiatric Illness. Elsevier: Academic Press: Amsterdam.

Ho C-Y (2010). The Ventral Pallidum as a Limbic Pleasure Generator. PhD Dissertation at The University of Michigan, Ann Arbor, MI, USA.

Jupp B, Krivdic B, Krstew E, Lawrence AJ (2011). The orexin(1) receptor antagonist SB-334867 dissociates the motivational properties of alcohol and sucrose in rats. Brain Res 1391: 54-59.

Kalivas PW, Nakamura M (1999). Neural systems for behavioral activation and reward. Cur Opin Neurobiol 9: 223-227.
Kelley AE, Baldo BA, Pratt WE (2005). A proposed hypothalamicthalamic-striatal axis for the integration of energy balance, arousal, and food reward. J Comp Neurol 493: 72-85.

Kim AK, Brown RM, Lawrence AJ (2012). The role of orexins/ hypocretins in alcohol use and abuse: an appetitive-reward relationship. Front Behav Neurosci 6: 78.

Kringelbach ML (2005). The human orbitofrontal cortex: linking reward to hedonic experience. Nat Rev Neurosci 6: 691-702.

Kupchik YM, Kalivas PW (2012). The rostral subcommissural ventral pallidum is a mix of ventral pallidal neurons and neurons from adjacent areas: an electrophysiological study. Brain Structure Function (doi:10.1007/s00429-012-0471-9).

Mahler SV, Smith KS, Berridge KC (2007). Endocannabinoid hedonic hotspot for sensory pleasure: anandamide in nucleus accumbens shell enhances 'liking' of a sweet reward. Neuropsychopharmacology 32: 2267-2278.

Mahler SV, Smith RJ, Moorman DE, Sartor GC, Aston-Jones G (2012). Multiple roles for orexin/hypocretin in addiction. Prog Brain Res 198: 79-121.

Marcus JN, Aschkenasi CJ, Lee CE, Chemelli RM, Saper CB, Yanagisawa $M$ et al (2001). Differential expression of orexin receptors 1 and 2 in the rat brain. J Comparative Neurol 435: 6-25.

Napier TC, Mickiewicz AL (2010). The role of the ventral pallidum in psychiatric disorders. Neuropsychopharmacology 35: 337-337.

Paxinos G, Watson C (1998). The rat brain in stereotaxic coordinates 4th edn. Academic Press: New York.

Peciña S, Berridge KC (2005). Hedonic hot spot in nucleus accumbens shell: Where do mu-opioids cause increased hedonic impact of sweetness? J Neurosci 25: 11777-11786.

Richard JM, Berridge KC (2011). Nucleus accumbens dopamine/ glutamate interaction switches modes to generate desire versus dread: $\mathrm{d} 1$ alone for appetitive eating but D1 and D2 together for fear. J Neurosci 31: 12866-12879.

Robinson MJF, Berridge KC. in press (2013). Instant transformation of learned repulsion into motivational 'wanting'. Curr Biol 23: $282-289$.

Shimura T, Imaoka H, Yamamoto T (2006). Neurochemical modulation of ingestive behavior in the ventral pallidum. Eur $J$ Neurosci 23: 1596-1604.

Smith KS, Berridge KC (2005). The ventral pallidum and hedonic reward: neurochemical maps of sucrose 'liking' and food intake. J Neurosci 25: 8637-8649.

Smith KS, Berridge KC (2007). Opioid limbic circuit for reward: interaction between hedonic hotspots of nucleus accumbens and ventral pallidum. J Neurosci 27: 1594-1605.

Smith KS, Berridge KC, Aldridge JW (2011). Disentangling pleasure from incentive salience and learning signals in brain reward circuitry. Proc Natl Acad Sci USA 108: E255-E264.

Smith KS, Mahler SV, Pecina S, Berridge KC (2010a). Hedonic Hotspots: Generating Sensory Pleasure in the Brain. In: Kringelbach MLBerridge KC eds. Pleasures of the Brain. Oxford University Press: Oxford, UK, pp 27-49.

Smith KS, Tindell AJ, Aldridge JW, Berridge KC (2009). Ventral pallidum roles in reward and motivation. Behav Brain Res 196: 155-167.

Smith RJ, Tahsili-Fahadan P, Aston-Jones G (2010b). Orexin/ hypocretin is necessary for context-driven cocaine-seeking. Neuropharmacology 58: 179-184.

Steiner JE (1973). The gustofacial response: observation on normal and anencephalic newborn infants. Symposium on Oral Sensation and Perception 4: 254-278.

Steiner JE, Glaser D, Hawilo ME, Berridge KC (2001). Comparative expression of hedonic impact: affective reactions to taste by human infants and other primates. Neurosci Biobehav Rev 25: 53-74.

Stoeckel LE, Weller RE, Cook EW 3rd, Twieg DB, Knowlton RC, Cox JE (2008). Widespread reward-system activation in obese 
Orexin hotspot in ventral pallidum

C-Y Ho and KC Berridge

women in response to pictures of high-calorie foods. Neuroimage 41: 636-647.

Stratford TR, Wirtshafter D (2012). Lateral hypothalamic involvement in feeding elicited from the ventral pallidum. Eur $J$ Neurosci 37: 648-653.

Thompson JL, Borgland SL (2011). A role for hypocretin/orexin in motivation. Behav Brain Res 217: 446-453.

Thompson RH, Swanson LW (2010). Hypothesis-driven structural connectivity analysis supports network over hierarchical model of brain architecture. Proc Natl Acad Sci USA 107: 15235-15239.

Thorpe AJ, Kotz CM (2005). Orexin A in the nucleus accumbens stimulates feeding and locomotor activity. Brain Res 1050: 156-162.

Tindell AJ, Smith KS, Berridge KC, Aldridge JW (2009). Dynamic computation of incentive salience: 'wanting' what was never 'liked'. J Neurosci 29: 12220-12228.

Tindell AJ, Smith KS, Pecina S, Berridge KC, Aldridge JW (2006). Ventral pallidum firing codes hedonic reward: when a bad taste turns good. J Neurophysiol 96: 2399-2409.
Valenstein ES, Cox VC, Kakolewski JW (1970). Reexamination of the role of the hypothalamus in motivation. Psychol Rev 77: 16-31.

Wassum KM, Ostlund SB, Maidment NT, Balleine BW (2009). Distinct opioid circuits determine the palatability and the desirability of rewarding events. Proc Natl Acad Sci 106: 12512-12517.

Zahm DS, Parsley KP, Schwartz ZM, Cheng AY (2013). On lateral septum-like characteristics of outputs from the accumbal hedonic 'hotspot' of Peciña and Berridge with commentary on the transitional nature of basal forebrain 'boundaries'. J Comp Neurol 521: 50-68.

Zahm DS, Williams E, Wohltmann C (1996). Ventral striatopallidothalamic projection: IV. Relative involvements of neurochemically distinct subterritories in the ventral pallidum and adjacent parts of the rostroventral forebrain. J Comp Neurol 364: 340-362.

Zheng H, Patterson L, Berthoud H (2007). Orexin signaling in the ventral tegmental area is required for high-fat appetite induced by opioid stimulation of the nucleus accumbens. J Neurosci 27: 11075-11082. 